\title{
Adaptive radiotherapy from past to future frontiers
}

\section{Introduction}

Adaptive radiation therapy (ART) is a radiation therapy process where treatment is adapted to account for internal anatomical changes which includes tumor shrinkage, weight loss or inflammation reduction. Certain organs-at-risk (OAR) in the body near to the region of interest for radiation therapy, can change in size and shape over the days and weeks during the course of treatment. The aim of ART is to account for these changes and deliver the radiation dose to the tumour as accurately as possible. With the emergence of the on boardvolumetric-imaging devices, the implication of ART has dramatically increased. For the first day of the treatment, an imaging and plan related algorithm leads to construct the patient's non-treatment geometric model. Thus, one can adaptively modify not only the PTV margin, but also the spatial dose distribution to best accommodate any change in patient's anatomy as well as the dosimetric deviation from the prescription incurred in previous fractions. A cumulative treatment plan provides an overall estimation of the treatment. In the last few years, much of the research has been dedicated to this ART and also notably, various vendors are making significant efforts. With technical advancements, particularly in computer, networking technology and optimization algorithms, online practices are becoming increasingly feasible and may likely become a "standard practice" in the near future. In this approach, the role of simulation geometry and the treatment plan is different from whatever is in current practice. In conclusion, the workflow and technology features are essential factors for introducing and providing robust adaptation of the treatment plan throughout the course of treatment. Indeed, the useful measure to identify patients' need for an adaptive treatment is the goal of strategy.

\section{First definitions for adaptive radiation therapy (ART)}

The concept of adaptive radiotherapy (ART) was formalized by Yan et al. ${ }^{1}$ as a radiotherapy process using systematic feedback of measurements by monitoring treatment variations during treatment of each individual patient. In order to further taking into account the changes in the geometry re-optimization of the treatment plan at each fraction become indispensable. This concept has been foreseeing the innovation that lead to clarifying of other treatment variations such as radiation sensitivity and density of clonogenic cells when they become measurable during the treatment course.

One of the major efforts in radiotherapy has been in reducing the effect of treatment variations related to geometric variables, such as displacement of radiation beam and treatment target and critical normal tissue and organs. ${ }^{1}$ Hence, related to the main subject i.e. implementation of ART process, the safe dose escalation and the optimization were considered at each involved clinic practice. This leads to the deeper analytic glance regarding margins and treatment doses, that can eventually be customized as per the daily needs of individual patients. The horizon extends to interpretations of individual optimization in margin and dose as well as the future concept of tumor control probability.

In a prospective clinical study by Di Yan et al. ${ }^{1}$ it was observed that they led the first adaptive study with the detection of possible setup errors in prostate cancer radiotherapy and its effect on the tumor dose. They mentioned that the ART is a feedback treatment process
Volume 8 Issue 2 - 202 I

\author{
Nina Tunçel \\ Science Faculty, Physics Department, Akdeniz University, Turkey \\ Correspondence: Nina Tunçel, Science Faculty, Physics \\ Department,Antalya,Turkey, Email ninatuncel@akdeniz.edu.tr \\ Received: December 25, 2020 | Published: May II, 202 I
}

according to patient's specific image information measured during the course of treatment by utilizing an imaging device. The electronic portal imaging device (EPID) was used in this study. The process was also equipped to be further extended to re-optimize the treatment plan by incorporating the predicted patient specific set-up variation was concluded. ${ }^{2}$

ART has been an active field of research during the last decade, and it is now being implemented in clinics by different applicable and practicable ideas. ${ }^{3}$ Essentially, ART process is progressively improved regarding the treatment regions by using newly established alternative technologies.

One of the major efforts in radiotherapy has been in reducing the effect of treatment variations not only related to machine but also to patient set up or organ motion. Some of the variations arise due to the mismatching of beam placement and immobilizing apparatus, and the others are geometrical disparity of treatment target and critical normal organs. The general validity of ART needs efficiently implemention process incorporating the actual variation of the individual patient. Generally, this forms the basis framework which mainly leads to setting the ART process; as implementation activities of the ART process, safe dose escalation in the ART and optimization of the ART process. Actually in the ART process, decision making on the optimal margins and treatment doses for intended target can be provided and customized for the individual patient. Optimization can then be more efficiently implemented incorporating to the actual variation of the individual patient.

Hence, the Tumor Control Probability (TCP) concept directly conjunctions to the ART process. On the other hand, it also leads to improve dramatically the Normal Tissue Control Probability (NTCP) concept as well. ${ }^{4,5}$ ART has been an active field essentially by scientific forces behind the medical process in radiotherapy during the last decades and it is now being implemented in clinics by merging different applicable ideas regarding the treatment regions by using newly established alternative technologies.

Utilization of an electronic portal imaging device (EPID) in many prospective clinical studies demonstrate that the predicted patient specific setup variation on 2-dimensional portal visualization have been a good practice in pursuance for management on ART process. ${ }^{2}$ Recently, the Image-Guidance Radiotherapy (IGRT) technologies are dominant in clinical practice by using 3 and 4-dimension visualization capabilities of different volumetric imaging devices. Therefore, the ART process has contained the off-line or on-line adjustment of the treatment delivery accordingly. 


\section{Basic components in ART}

Generally, the adaptive radiotherapy system consists of five basic components. The title and related purposes of each component that are recommended by $\operatorname{Yan}^{6}$ are briefly shown in Table 1 . The value of ART may not only be restricted to the adaptation of treatment volumes during radiotherapy, but may also be used to define and evaluate which patients can benefit from dose escalation during treatment. ${ }^{7}$ The lack of sufficient information regarding patient selection for applying ART was mentioned by Brouwer et al. in head and neck radiotherapy. ${ }^{8}$ Identifying patients who may get benefit from ART needs very large data collection related to both regional anatomic site modifications and dosimetric effects on PTV and organsatrisk in each selected carcinoma.

Table I Basic Component in ART

\begin{tabular}{lll}
\hline Component & Title & Subject \\
\hline I & $\begin{array}{l}\text { Treatment delivery } \\
\text { to deliver radiation dose to the patient based on a treatment plan } \\
\text { to observe and verify patient/organ temporal variation before, during, and/or after the } \\
\text { treatment delivery } \\
\text { to estimate, based on image feedback, the parameters which can characterize the undergoing } \\
\text { temporal variation process, and evaluate the corresponding treatment parameters, such as } \\
\text { the cumulative dose, biological effective dose, TCP, NTCP, etc. }\end{array}$ \\
& $\begin{array}{l}\text { Estimation/ Evaluation } \\
\text { Design of adaptive } \\
\text { planning /adjustment }\end{array}$ & $\begin{array}{l}\text { to design and update planning/adjustment parameters, as well as modify imaging, delivery, and } \\
\text { adjustment schedules, in response to the estimation and the evaluation } \\
\text { to perform a 4D conformal or IMRT planning with the usage of planning parameters } \\
\text { specified in the adaptive planning/ adjustment design, and adjust treatment delivery } \\
\text { accordingly }\end{array}$ \\
\hline
\end{tabular}

The sub title on basic components in ART are mainly link to selection or judgment properties during image acquisition that offer the off-line, on-line and in-line workflow. On the other hand, the software that is planned to use will be compatible to ruling adjustments regarding to re-planning on implementing of ART.

\section{IG technologies}

Image-guidance (IG) technologies were designed to improve the targeting of tumour tissues while reducing exposure to normal tissues using radiation beams that could be historically categorized regarding technologies. It will allow physicians to precisely locate a tumour, as well as lock onto it during delivery, even when tumour tissue is moving during treatment or changes shape, location or size between treatment sessions. The IG technologies have been improved and generally the prominent products have been manufactured into three main generations (Table 2).

\section{Adaptive radiotherapy (ART) strategies}

The clinical implementation mission of every new technology such as ART is to ensure the technical confirmation by retaining a specific quality assurance steps with utmost safety. ART has been anticipated to customize the treatment either to the motion or the response pattern of the individual patients by re-planning strategies, but the whole process increases the workload and thereby challenges the clinical practice. Adaptation may be performed on-line, off-line or in a hybrid manner. The newer set of images (essentially CT scan) is required and other images PET or MR can be taken as desired. Onto these new images of patients the radiationoncology team needs to perform many processes starting with image registration, segmentation, planning on the adaptive CT scan and finally evaluation of the cumulative dose. Fractional and cumulative dose evaluations are based on Monte-Carlo calculation also sometimes use of deformable image registration methods or more complex methods are employed. Hence, the increased dependence on new technologies and on more available specialized human resources have actualized on implementing ART.

Table 2 Image-guidance (IG) technologies

\begin{tabular}{|c|c|}
\hline Generation & Prominent Products \\
\hline & $\begin{array}{l}\text { Ultrasound (US), kV Radiographic, Portal imaging, and } \\
\text { Markers (active and passive) }\end{array}$ \\
\hline \multicolumn{2}{|l|}{ I } \\
\hline & kV-CT approach as Siemens PRIMATOMTM, \\
\hline \multirow[t]{4}{*}{ II } & MV-CT approach as Tomotherapy Hi-ArtTM, \\
\hline & $\begin{array}{l}\text { kV Cone-beam CT (CBCT) approach as Elekta } \\
\text { SynergyTM and Varian OBITM, }\end{array}$ \\
\hline & $\begin{array}{l}\text { MV Cone-beam CT approach as Siemens MVisionTM and } \\
\text { ArtisteTM }\end{array}$ \\
\hline & US++ approach as Clarity system, \\
\hline \multirow[t]{2}{*}{ III } & $\mathrm{kV}-\mathrm{CBC} T++$ and conjunction with $\mathrm{kV}-\mathrm{kV}$ approach \\
\hline & $\begin{array}{l}\text { MR-Guided RT as Elekta-Philips Linac approach,ViewRay's } \\
\text { MRIdian Linac and MagnetTx Oncology Solutions Aurora } \\
\text { RT product }\end{array}$ \\
\hline
\end{tabular}

The adaptive radiotherapy strategies for different tumor sites have been enrolled in number of studies. The benefit of pre-treatment planning library-based and forward re-planning are also discussed in literature for certain tumor sites. However, the latest application in routine clinical practice is limited due to the demanding nature of the processes that involved (re-delineation and re-planning) and hence to increase dependence on available human resources.

ART can be categorized based on the time scales roughly as off-line, ${ }^{2}$ on-line, and ${ }^{3}$ in-line. Typically, off-line adaptive plan 
adjustments are applied once during the course, or even on certain patients whose setups reveal large systematic errors. More common applications include the weekly adaptations of the plan have also been suggested.

Table 3 Strategies for adaptive radiotherapy

Current ART approaches and Clinical implementation of ART
Off-line adaptation On-line adaptation $\quad$ In-line adaptation
PTV \& OAR modification
PTV modification based on patient setup errors
PTV modification based on internal organ motion tumor and nodal
regression
OAR modification based on volumetric and positional changes or weight
loss
-
Imaging techniques for implementation of ART
In-room imaging for ART
Fan beam CT imaging
Cone-beam CT imaging
MR imaging
Other imaging modalities
Challenges to implementation of ART as per each site / type cancer.

On-line adaptive protocols typically are applied daily, and they not only use the image information of previous fractions to adapt the treatment plan but also an image acquired during the fraction itself Thus, on-line protocols have the ability to correct for inter fraction and day-to-day variations.

Magnetic Resonance-guided radiotherapy (MRgRT) provides real-time information for in-line adaptive protocols. MRI navigator signals offer high time-based data on 1-dimensional (1D) motion and have been assessed for both gated deliveries by real-time tracking. On the other hand, several orthogonal cine MR images with low latency are defined to track the target in 3D. ${ }^{9}$ The recent technological developments of MRgRT in commercial platform provides abundant opportunities for direct imaging in soft tissue contrast during the course of radiation treatment and in the treatment position, without adding imaging radiation dose to the patient. ${ }^{10}$

Two systems are initialized in working on clinical practice as Unity system (Elekta, Sweden) and MRIdian system (Viewray, USA). ${ }^{11,12}$ The MRgRT properties lead to MR-guided adaptive radiotherapy (MRgART), which includes MR-guided radiation delivery with on-line treatment plan adaptations to match daily anatomy. It is the early convention of on-line treatment plan adaptation that forcing to reconsider paradigms of radiation therapy and to re-imagine the clinical workflows. ${ }^{10}$

MRI offers the ability to image biological and functional aspects of the body. Therefore, MRI has the potential to provide imaging biomarkers of therapy response of tumor or normal tissue or both. Subsequently, these imaging biomarkers can drive adaptive plan modifications to account for the observed therapy response as Biology-guided adaptive radiotherapy. ${ }^{9}$ The early results were different for each treatment site and indicating that online treatment plan evaluation should have site-specific protocols. ${ }^{13}$
Image registration based on deformable image registration (DIR) algorithms could be used and lead to a reduction in both work and calculation time with on-line strategy. Also it can be used as "offline" or "hybrid off-line \& on-line" strategies in time consumption and therapeutic gain for the patient. ${ }^{14}$ DIR for contour propagation and dose calculation would be mainly used for ART in terms of the delivered dose. ${ }^{15}$

Typically, off-line adaptive plan modifications are applied once during the treatment course, or even on selected patients whose setups demonstrate large systematic errors. On-line adaptive protocols would typically be applied daily, and such protocols not only use the image information of previous fractions to adapt the treatment plan but also an image acquired during the fraction itself. In-line ART protocols are an important development utilizing MRI to provide a real- time information. $^{9}$

In the last decade, researchers have made a great deal of effort to develop algorithms to speed up the re-planning process especially regarding ART implementation. Deep learning methods for image guidance in radiation therapy have been under progress to highlight tracks toward ART. ${ }^{16,17}$

However, its application in routine clinical practice is limited due to the demanding nature of the processes involved (re-delineation and re-planning) and increased dependence on the available human resources. The re-planning options range from simple adaptations, such as adjustments of beam weights, to complete re-planning that involves re-optimization of the intensity modulation.

\section{Future considerations}

Adaptive radiotherapy strategies will give us a vast amount of information related to the merit features in BiGART (Biological Guided Adaptive Radiotherapy) that the entire radiation oncology community is looking at. Findings that shed new light on the possible imaging toward biological processing in the course of ART leads to the use of either PET or MRI in room. This will assist the entire radiation oncology community to narrow down the research for establishing new processing on the acquisition of images and altering the clinical practice accordingly.

Such vital researches and advances in the clinical practice will actually improve the patient outcomes with rational recommendations. Hence, the evidence-based recommendations will give new horizon to realize the future of BiGART processes. The deep learning-based algorithms offer sufficient opportunities to solve the problem of typically handling the required large imaging datasets in the ART process. Improving the deep learning performance is clinically relevant with its applications ranging from the initial contouring process to the in-line adaptive radiotherapy strategies.

\section{Acknowledgment}

None.

\section{Conflicts of interest}

Author declares there are no conflicts of interest.

\section{Funding}

None. 


\section{References}

1. Yan D, Vicini F, Wong J, et al. Adaptive radiation therapy, Phys Med Biol. 1997;42(1):123-32.

2. Yan D, Ziaja E, Jaffray D, et al. The use of adaptive radiation therapy to reduce setup error: a prospective clinical study. Int. J. Radiation Oncology Biol Phys. 1998;41(3):715-720.

3. Tuomikoski L, Valli A, Tenhunen M, et al. A comparison between two clinically applied plan library strategies in adaptive radiotherapy of bladder cancer, Radiother Oncol. 2015;117(3):448-452.

4. Ten Eikelder SCM, Ferjančič P, Ajdari A, et al. Optimal treatment plan adaptation using mid-treatment imaging biomarkers. Phys Med Biol. 2020;65(24):245011.

5. Heukelom J, Kantor ME, Mohamed ASR, et al. Differences between planned and delivered dose for head and neck cancer, and their consequences for normal tissue complication probability and treatment adaptation. Radiother Oncol. 2020;142:100-106.

6. Yan D. Image-Guided/Adaptive Radiotherapy. In: Schlegel W, Bortfeld T, Grosu AL. (eds) New Technologies in Radiation Oncology. Medical Radiology (Radiation Oncology). Springer, Berlin, Heidelberg. 2006.

7. Pierre Castadot, John Alee, Xavier Geets, et al. Adaptive radiation therapy for head and neck cancer. Applied Radiat Oncol. 2010;20(2):8493.

8. Brouwer CL, Steenbakkers RJ, Langendijk JA, et al. Identifying patients who may benefit from adaptive radiotherapy: Does the literature on anatomic and dosimetric changes in head and neck organs at risk during radiotherapy provide information to help? Radiother Oncol. 2015;115(3):285-294.
9. Kupelian P, Sonke JJ. Magnetic resonance-guided adaptive radiotherapy: a solution to the future. Semin Radiat Oncol. 2014;24(3):227-232.

10. Landry G, Corradini S, Belka C. Magnetic resonance-guided radiation therapy: the beginning of a new era. Radiat Oncol. 2020;15(1):163.

11. Wen N, Cao Y, Cai J. Editorial: Magnetic Resonance Imaging for Radiation Therapy. Front Oncol. 2020;10:483.

12. Kurz C, Buizza G, Landry G, et al. Medical physics challenges in clinical MR-guided radiotherapy. Radiat Oncol. 2020;15(1):93.

13. van Timmeren JE, Chamberlain M, Krayenbuehl J, et al. Treatment plan quality during online adaptive re-planning. Radiat Oncol. 15(1):203.

14. Henriques de Figueiredo B, Petit A, Sargos P, et al. Radiothérapie adaptative en routine: point de vue de l'oncologue radiothérapeute. Cancer Radiother. 2015;19(6-7):446-449.

15. Rigaud B, Simon A, Castelli J, et al. Deformable image registration for radiation therapy: principle, methods, applications and evaluation. Acta Oncol. 2019;58(9):1225-1237.

16. Paysan P. et al. Deep Learning Methods for Image Guidance in Radiation Therapy. In: Schilling FP., Stadelmann T. (eds) Artificial Neural Networks in Pattern Recognition. ANNPR 2020. Lecture Notes in Computer Science, vol 12294. Springer, Cham.

17. Boldrini L, Bibault JE, Masciocchi C, et al. Deep Learning: A Review for the Radiation Oncologist. Front Oncol. 2019;9:977. 